\title{
Characterizing the metabolic heterogeneity in human breast cancer xenografts by 3D high resolution fluorescence imaging
}

He N Xu ${ }^{1,2}$, Gang Zheng ${ }^{3}$, Julia Tchou ${ }^{4,5}$, Shoko Nioka ${ }^{2}$ and Lin Z Li $i^{1,2^{*}}$

\begin{abstract}
We previously reported that tumor mitochondrial redox state and its heterogeneity distinguished between the aggressive and the indolent breast cancer xenografts, suggesting novel metabolic indices as biomarkers for predicting tumor metastatic potential. Additionally, we reported that the identified redox biomarkers successfully differentiated between the normal breast tissue and the cancerous breast tissue from breast cancer patients. The aim of the present study was to further characterize intratumor heterogeneity by its distribution of mitochondrial redox state and glucose uptake pattern in tumor xenografts and to further investigate the metabolic heterogeneity of the clinical biopsy samples. We employed the Chance redox scanner, a multi-section cryogenic fluorescence imager to simultaneously image the intratumor heterogeneity in the mitochondrial redox state and glucose uptake at a high spatial resolution (down to $50 \times 50 \times 20 \mu^{3}$ ). The mitochondrial redox state was determined by the ratio of the intrinsic fluorescence signals from reduced nicotinamide adenine dinucleotide (NADH) and oxidized flavoproteins (Fp including FAD, i.e., flavin adenine dinucleotide), and the glucose uptake was measured using a near-infrared fluorescent glucose-analogue, pyropheophorbide 2-deoxyglucosamide (Pyro-2DG). Significant interand intratumor metabolic heterogeneity were observed from our imaging data on various types of breast cancer xenografts. The patterns and degrees of heterogeneity of mitochondrial redox state appeared to relate to tumor size and metastatic potential. The glucose uptake was also heterogeneous and generally higher in tumor peripheries. The oxidized and reduced regions mostly corresponded with the lower and the higher pyro-2DG uptake, respectively. However, there were some regions where the glucose uptake did not correlate with the redox indices. Pronounced glucose uptake and high NADH were observed in certain localized areas within the tumor necrotic regions, indicative of the existence of viable cells which was also supported by the H\&E staining. Significant heterogeneity of the redox state indices was also observed in clinical specimens of breast cancer patients. As abnormal metabolism including the Warburg effect (high glycolysis) plays important roles in cancer transformation and progression, our observations that reveal the 3D intratumor metabolic heterogeneity as a characteristic feature of breast tumors are of great importance for understanding cancer biology and developing diagnostic and therapeutic methods.
\end{abstract}

Keywords: The Chance redox scanner, Redox scanning, Glucose uptake, NADH, Flavoprotein (Fp), FAD, Mitochondrial redox state, Pyro-2DG, MCF-7, MDA-MB-231, MDA-MB-468

\footnotetext{
* Correspondence: linli@mail.med.upenn.edu

${ }^{1}$ Molecular Imaging Laboratory, Department of Radiology, Perelman School of Medicine, University of Pennsylvania, Pennsylvania, USA

${ }^{2}$ Britton Chance Laboratory of Redox Imaging, Johnson Research Foundation, Department of Biochemistry and Biophysics, Perelman School of Medicine, University of Pennsylvania, Pennsylvania, USA

Full list of author information is available at the end of the article
}

\section{实 Springer}

(c) 2013 Xu et al. This is an Open Access article distributed under the terms of the Creative Commons Attribution License (http://creativecommons.org/licenses/by/2.0), which permits unrestricted use, distribution, and reproduction in any medium, provided the original work is properly cited. 


\section{Introduction}

Abnormal metabolism and high variability in disease presentations are among the hallmarks of cancer (Heppner \& Miller 1998; Hanahan \& Weinberg 2011). The intratumor heterogeneity or diversity has been associated with tumor progression and aggressiveness (Heppner 1984; Allred et al. 2008; Park et al. 2010). It is therefore of great importance to characterize tumor metabolic heterogeneity.

Cancer metabolism has been an active research area for understanding tumor biology and developing biomarkers for cancer diagnosis and monitoring treatment response (Cairns et al. 2011; Vander Heiden et al. 2009). Higher than normal glucose uptake/metabolism as first identified by Otto Warburg in 1920s (Koppenol et al. 2011), lays the foundation for cancer staging by fluorinated 2-deoxyglucose positron emission tomography (FDG-PET) in the clinic. Studies also investigated the glucose uptake and its spatial heterogeneity in animal models (Kallinowski et al. 1988; Dearling et al. 2004). Another area under active research investigation is mitochondrial metabolism including TCA cycle and oxidative phosphorylation. Several mitochondrial metabolic enzymes have been identified as oncogenes or tumor suppressors in some cancers (Thompson 2009).

Previously we reported that the mitochondrial redox state and its heterogeneity in tumors imaged by the Chance redox scanner provide sensitive and potentially diagnosis-useful indices for differentiating among five human melanoma lines and two breast cancer lines with different metastatic potential in mouse models ( $\mathrm{Li}$ et al. 2009a; Xu et al. 2010). In addition, we reported that the redox imaging indices differentiated between the normal and cancerous breast tissues from breast cancer patients (Xu et al. 2013a). We showed that the aggressive or metastatic tumors have localized more oxidized areas (higher Fp redox ratio, $\mathrm{Fp} /(\mathrm{Fp}+\mathrm{NADH})$ ), and that the Fp redox ratios of the oxidized areas positively correlated with tumor metastatic potential and thus could be further developed for grading tumor aggressiveness of human melanoma and breast cancer. The pattern of the oxidized areas with surrounding reduced areas appears to be a distinct feature of intratumor heterogeneity in aggressive tumors. However, the 3D heterogeneity of the mitochondrial redox indices (NADH, Fp, and the Fp redox ratio) has not been thoroughly investigated for whole tumors.

Because of cancer cells' higher than normal glycolysis, simultaneously imaging the glucose uptake/metabolism and the mitochondrial redox state in tumors adds one more dimension to our understanding of tumor metabolism. As the size of an oxidized tumor area can be as small as $1-2 \mathrm{~mm}$ (Xu et al. 2010), FDG-PET is not suitable for such a purpose due to its low spatial resolution (1-2 mm for small animals and $\sim 4 \mathrm{~mm}$ for human subjects). Previously, Zheng and co-workers developed the near-infrared fluorescent glucose-analog Pyro-2DG and also confirmed that Pyro-2DG was selectively accumulated in tumor cells via the glucose transporters (Zhang et al. 2003). Using the high-resolution Chance redox scanner, they achieved simultaneous imaging of Pyro-2DG uptake and the redox indices of the 9L glioma and c-MYC-induced mammary tumors in animal models (Zhang et al. 2004). The imaging data showed that Pyro-2DG uptake correlated well with the reduced redox state and did not affect the redox state of the tumor tissue.

In the present study, we first report the 3D distributions of the mitochondrial redox state in the three breast tumor xenografts in nude mice. We also report the significant intra-tumor heterogeneity of the mitochondrial redox state observed in tumor samples from breast cancer patients. We then show the glucose uptake distribution pattern and its correlations with the redox indices in the breast tumor xenografts. A portion of the preliminary data from mouse models were published in a conference proceeding (Xu et al. 2012; Xu et al. 2011a). The results are useful for deepening our understanding on tumor metabolism and its heterogeneity, which may drive the development of novel diagnostic and therapeutic methods for cancer management.

\section{Materials and methods}

\section{Tumor models and sample preparation for redox} scanning

The animal protocols were approved by the Institutional Animal Care and Use Committee at the University of Pennsylvania. Subcutaneous xenografts of three human breast cancer cell lines with increasing metastatic potential (MCF-7 < MDA-MB-468 < MDA-MB-231, N $\geq 5$ for each line) were grown to $6-10 \mathrm{~mm}$ in diameter in athymic nude mice for the 3D redox scanning. Another two MDAMB-231 tumor xenografts $(>10 \mathrm{~mm}$ in diameter and with an apparent necrotic center, average tumor volume $=$ $\left.989 \mathrm{~mm}^{3}\right)$ and two MCF-7 tumor xenografts $(>10 \mathrm{~mm}$ in diameter, average tumor volume $=750 \mathrm{~mm}^{3}$ ) were used for simultaneous imaging of Pyro-2DG uptake and the redox state. Pyro-2DG was administered to the anesthetized mice through tail vein injection at a dosage of $\sim 2.5 \mathrm{mg} / \mathrm{kg}$ after the tumor-bearing mice were starved for $24 \mathrm{hrs}$. In approximately two hours, the anesthetized mice were snap-frozen using liquid nitrogen. The snap-freezing protocol maintained the in vivo mitochondrial redox state for the ex vivo redox scanning. The preparation of tissue samples for the redox scanning were previously described (Xu et al. 2010; Xu et al. 2009a). Briefly, the excised frozen samples were embedded with chilled mounting buffer (ethanol:glycerol:water $=10: 30: 60$ ). Frozen reference standards of NADH, FAD, and Pyro-2DG with known concentrations (in $10 \mathrm{mM}$ Tris- $\mathrm{HCl}$ buffer, $\mathrm{pH} \sim 7$ ) were quickly mounted adjacent to the tissue for the purpose of 
calibrating the concentrations of these fluorescent molecules in tissue.

\section{Human breast tumor tissue collection}

Tissue collection from patients was performed according to a protocol approved by the Internal Review Board of the University of Pennsylvania. Both normal and cancerous tissue specimens were collected from the cancerbearing breasts of three patients shortly after surgical resection. Core biopsies and/or thin tissue blocks were obtained from tumor and normal adjacent breast tissue, respectively and were snap-frozen in liquid nitrogen within 5-15 minutes after the tissue removal from the body. These tissue specimens were embedded in the same way as described for the xenografts aforementioned.

\section{Redox scanning using the chance redox scanner}

The low-temperature redox scanner developed by Chance et. al (Chance et al. 1979; Gu et al. 2002; Li et al. 2009b; Quistorff et al. 1985) (the Chance redox scanner) was employed to obtain the multi-slice fluorescence images of NADH, Fp, and Pyro-2DG. The optical filters were $430 \mathrm{~nm} \pm 25 \mathrm{~nm}$ and $525 \mathrm{~nm} \pm 32 \mathrm{~nm}$ for Fp excitation and emission channel respectively; $360 \mathrm{~nm} \pm 13 \mathrm{~nm}$ and $430 \mathrm{~nm} \pm 25 \mathrm{~nm}$ for NADH excitation and emission channel, respectively; and $430 \pm 28 \mathrm{~nm}$ and $670 \pm 11 \mathrm{~nm}$ for pyro-2DG excitation and emission, respectively. For the 3D imaging study, the tumor samples were scanned section by section at different tissue depths with 400 $800 \mu \mathrm{m}$ spacing. Most tumors were scanned at least to the center. For some tumors the entire tumor was scanned. For the study of simultaneous imaging of NADH, Fp, and pyro-2DG, the fluorescent signals were obtained from 2
3 tissue sections with depth ranging from $\sim 1700-3000 \mu \mathrm{m}$ under the mouse skin.

\section{Data analysis}

The nominal concentration of NADH, Fp, and pyro-2DG in tissue was interpreted using the fluorescence intensity of the corresponding reference standard, respectively and further used to calculate $\mathrm{Fp} /(\mathrm{NADH}+\mathrm{Fp})$ or $\mathrm{NADH} /$ $(\mathrm{Fp}+\mathrm{NADH})$. To account for the cell or mitochondria density difference at different regions within a tumor, the normalized Pyro-2DG, i.e. Pyro-2DG/(NADH + Fp) was also calculated based on the nominal concentrations of NADH and Fp.

In order to obtain the mean values in the more oxidized regions (high $\mathrm{Fp}$ redox ratio or $\mathrm{Fp} /(\mathrm{NADH}+\mathrm{Fp})$ ) and less oxidized regions (low Fp redox ratio), the sum of two Gaussian functions $f(x)=a_{1} e^{-\frac{\left(x-b_{1}\right)^{2}}{2 c_{1}{ }^{2}}}+a_{2} e^{-\frac{\left(x-b_{2}\right)^{2}}{2 c_{2}{ }^{2}}}$ was fitted to the histograms of the $\mathrm{NADH}, \mathrm{Fp}$, and Fp redox ratio images (Xu et al. 2013b). The fitted $b_{i}(i=1,2)$ values were taken as the means of these redox indices in the corresponding more or less oxidized region.

\section{Results}

3D redox images show high degree intertumor and intratumor heterogeneity

We have observed significant inter- and intratumor heterogeneity in the mitochondrial redox state in the breast cancer mouse xenografts. As the representative tumors shown in Figure 1, both aggressive lines (MDA-MB-231 and MDA-MB-468) displayed high degree of heterogeneity of $\mathrm{NADH}, \mathrm{Fp}$ and the Fp redox ratio in each section, with localized areas (usually tumor central regions)

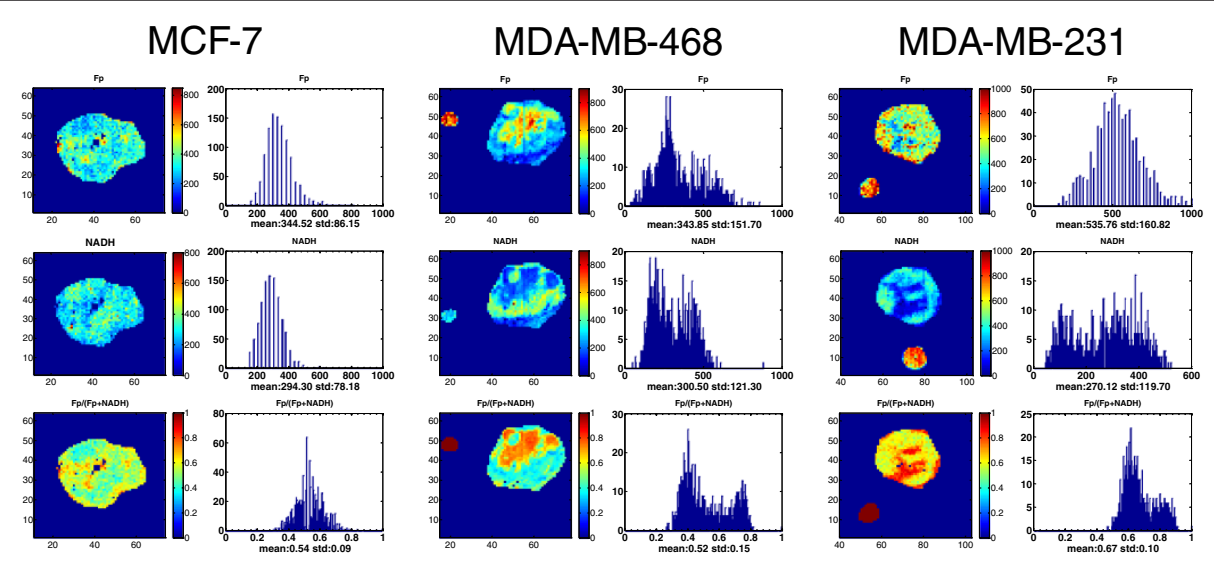

Figure 1 Typical images of redox scanning of the mouse xenografts of MCF-7 (left, depth $d=3400 \mu \mathrm{m}$ ), MDA-MB-468 (middle $\mathbf{d}=\mathbf{1 2 0 0} \boldsymbol{\mu m}$ ), and MDA-MB-231 (right, $\mathbf{d}=\mathbf{8 0 0} \boldsymbol{\mu m}$ ) tumor lines. The Fp or NADH redox ratio ranges between 0 and 1; the Fp or NADH images are in the unit of $\mu \mathrm{M}$ in reference to the corresponding standards. The $\mathrm{x}$ axes of the corresponding histograms represent the $\mathrm{Fp}$ redox ratio or concentration. The y axes represent the number of pixels in the tumor section having a specific value of Fp redox ratio or Fp and NADH concentration. The small round spots outside the tumor section are the images of Fp or NADH reference standards. The images are labeled with numbers on the bottom and left axes to indicate the positions of the image matrices with a spatial resolution of $200 \mu \mathrm{m}$. 
exhibiting much higher Fp redox ratio than the other regions (usually tumor peripheries). A bimodal distribution is observed in their histograms. The indolent MCF-7 tumors $(\leq 10 \mathrm{~mm}$ ) were relatively less heterogeneous in each section, with a lower Fp redox ratio on average than that of the oxidized regions of the aggressive tumors. However, as we will show below, the MCF-7 tumors with larger size also showed high degree of heterogeneity with a clear center-periphery pattern.

To see how the redox indices change along the depth of each tissue section, they were plotted against the depth of each tumor section of the three tumors in Figure 1 to demonstrate how the redox indices distribute globally within a tumor (Figure 2). The left column of Figure 2 contains the plots of section average value of each of the redox indices versus depth. It is clearly seen that all three redox indices vary with depth for each tumor line. The aggressive tumors appear to have higher variations than the less aggressive MCF-7, demonstrating their higher degree of heterogeneity along section depth in addition to that exhibited on each section. We can see that the most aggressive tumor MDA-MB-231 has the highest Fp and the $\mathrm{Fp}$ redox ratio values, indicating it is the most oxidized tumor.

In addition to the section average values, for the aggressive tumors, we also obtained the mean values of the redox indices of the core (defined as high Fp redox ratio regions) and those of the rim (defined as low Fp redox ratio regions) by fitting of two Gaussian functions to their corresponding histograms. The plots in the middle column of Figure 2 are for the MDA-MB- 468 tumor. We can see that the core and the rim are clearly separated for all three redox indices in the entire range of tissue depth. Fp is higher in the core area but drops sharply at $2200 \mu \mathrm{m}$ and similar pattern is seen for Fp in the rim area. NADH distributions are roughly opposite to those of Fp with the maxima at $1900 \mu \mathrm{m}$. The Fp redox ratio is markedly higher in the core than in the rim. In comparison, the redox indices of the core and rim of the MDA-MB-231 tumor exhibited a different depth-variation pattern, but had a similar separation between the rim and core except at one depth point for NADH (Figure 2, right column). All these patterns of the aggressive tumors can be characterized by a bimodal distribution of the mitochondrial redox state with a more and a less oxidized region.

Figure 3 displays the typical redox images of a breast cancer tissue block from a patient, who had an ER positive (>90\%) invasive lobular carcinoma (ILC) and treated by arimidex for close to 8 months before her mastectomy. The heterogeneity is readily spotted from the images and also evidenced from the large standard deviations shown on the histograms. Heterogeneity of the redox indices was also evident from each of the core biopsy specimens of the cancerous tissue (Xu et al. 2013a).

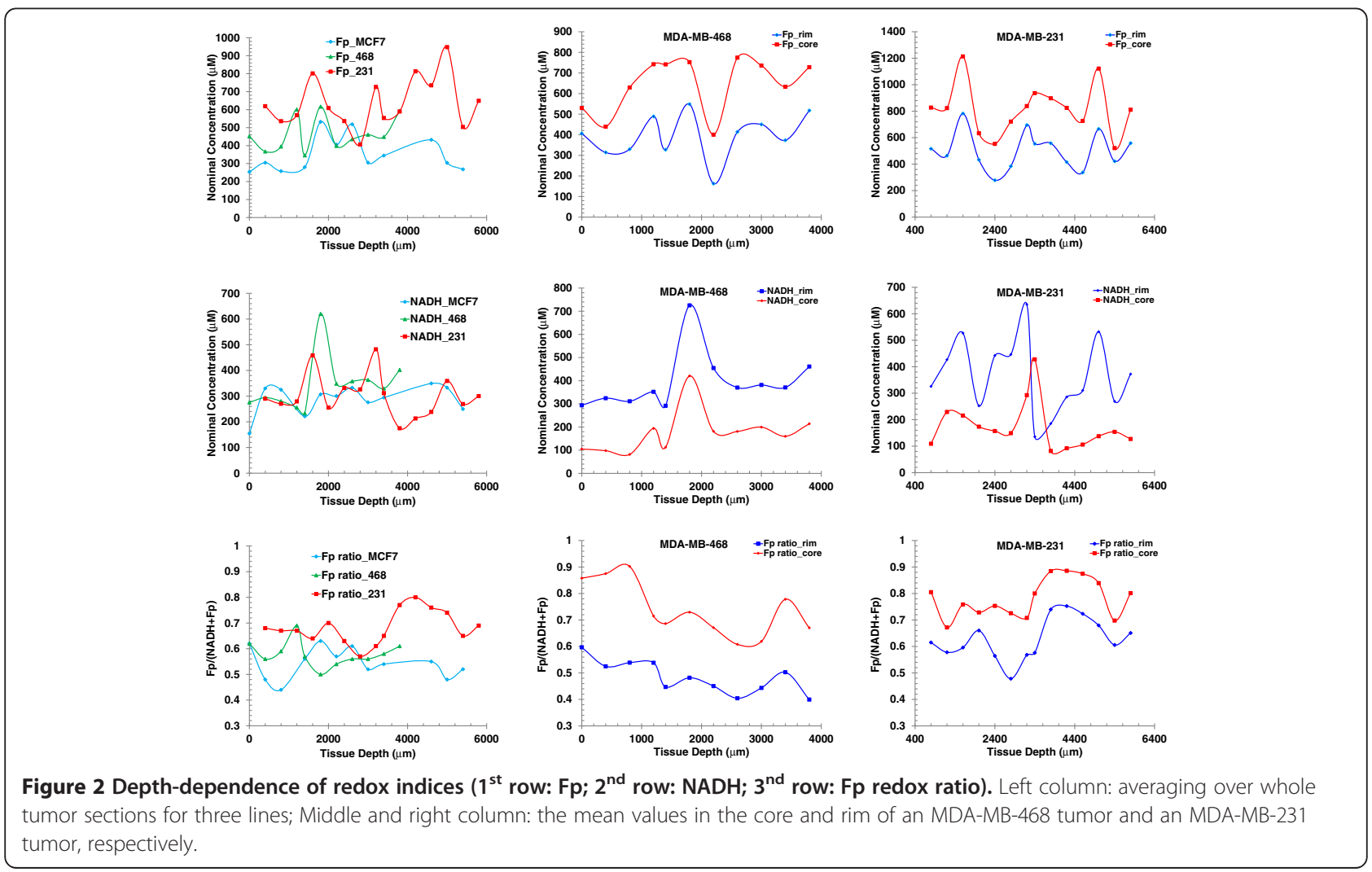



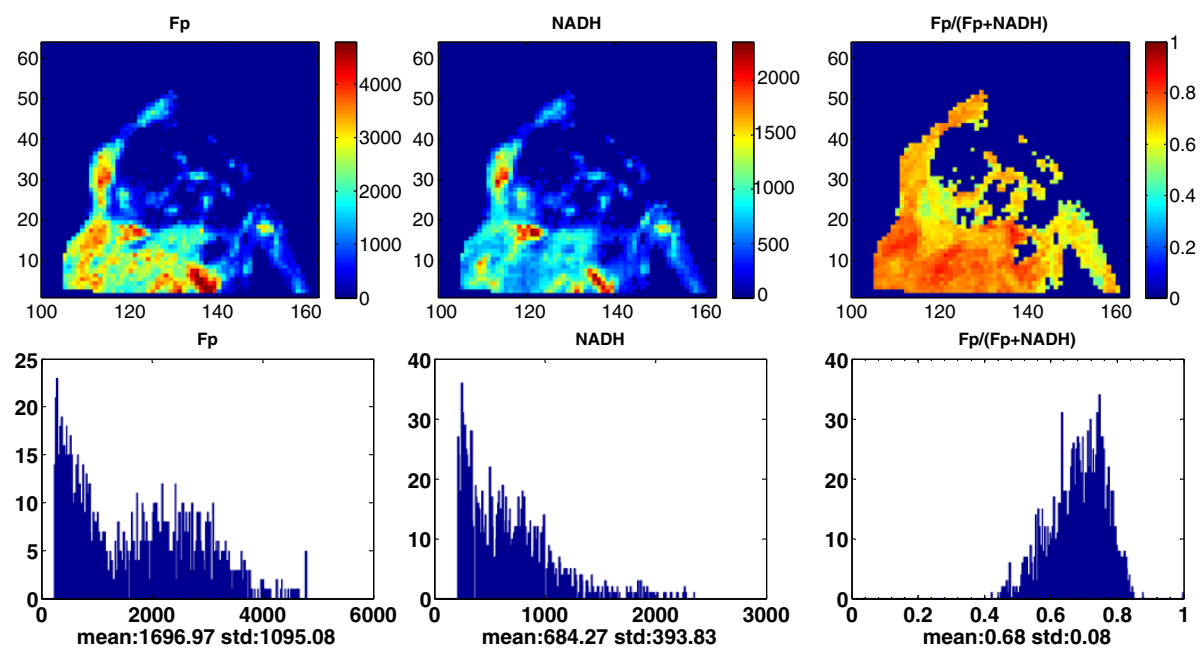

Figure 3 Typical mitochondrial redox images and the corresponding histograms of breast tumor tissue from a patient. Image matrix $256 \times 64$ (only partially displayed) and spatial resolution $100 \mu \mathrm{m}$.

Simultaneous imaging of Pyro-2DG uptake and the redox state reveals islands of viable cells existed in the necrotic centers

Figure 4 \& 5 show the images of $\mathrm{Fp}, \mathrm{NADH}$, and pyro2DG simultaneously acquired for two representative large and necrotic tumors of MCF-7 and MDA-MB-231, respectively. Also shown in these figures are the redox ratio images and those of pyro-2DG/(Fp + NADH), which are less sensitive to cell density variations by normalizing the concentrations to $\mathrm{Fp}+\mathrm{NADH}$. We again observed significant intra-tumor heterogeneity of the redox state and glucose uptake in both tumors, and their corresponding histograms also exhibited a bimodal or multi-modal distribution. Note that the large MCF-7 tumors had a core-rim pattern as the MDA-MB-231 tumors, which is not usually observed for smaller MCF7 tumors $(\leq 10 \mathrm{~mm})$.

Due to complete cell death, we can identify certain central regions where there were no fluorescence signals in all channels. However, within these necrotic centers,
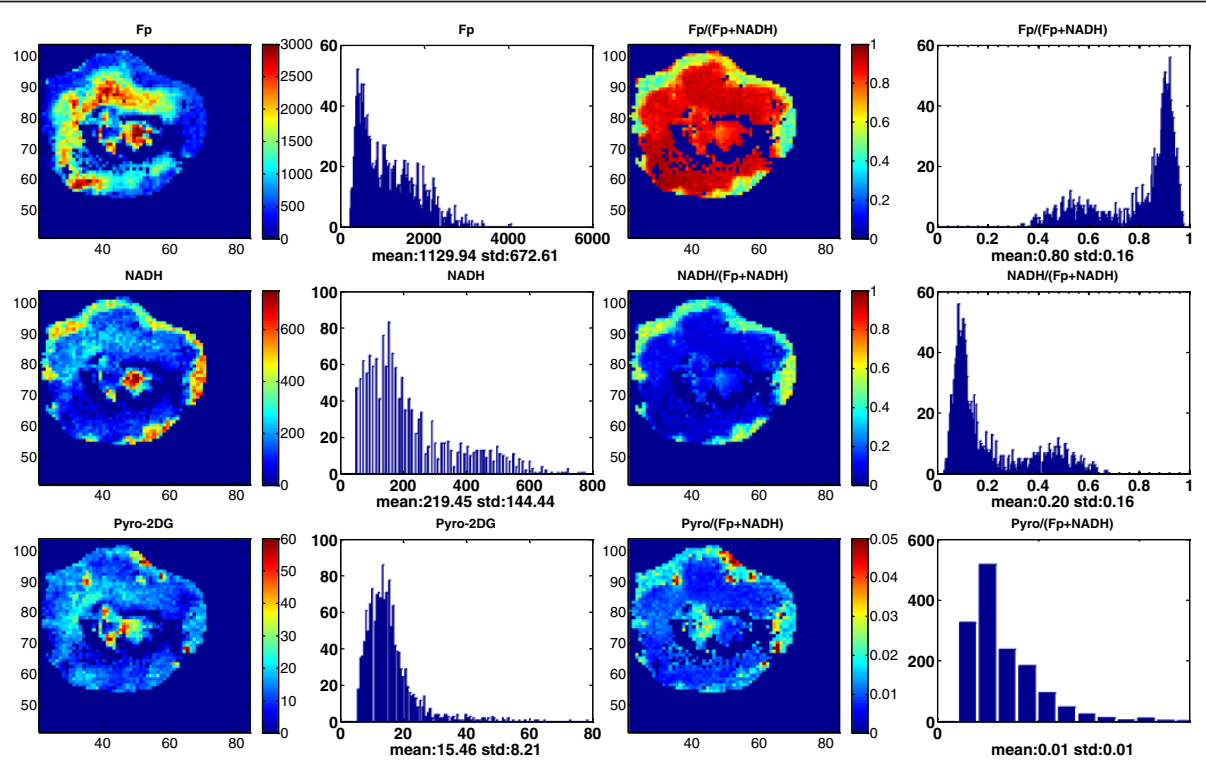

Figure 4 Typical mitochondrial redox state and Pyro-2DG uptake images and their corresponding histograms of a representative section of a large MCF-7 tumor $\left(>10 \mathrm{~mm}\right.$, Vol $=\mathbf{5 6 8} \mathrm{mm}^{3}$ ). The Fp redox ratio, NADH redox ratio, and normalized Pyro-2DG (Pyro-2DG/ $(\mathrm{Fp}+\mathrm{NADH})$ ) range between 0 and 1. The $\mathrm{Fp}, \mathrm{NADH}$, and Pyro-2DG images are in the unit of $\mu \mathrm{M}$ in reference to the corresponding standards. For other notations see the legend of Figure 1. 

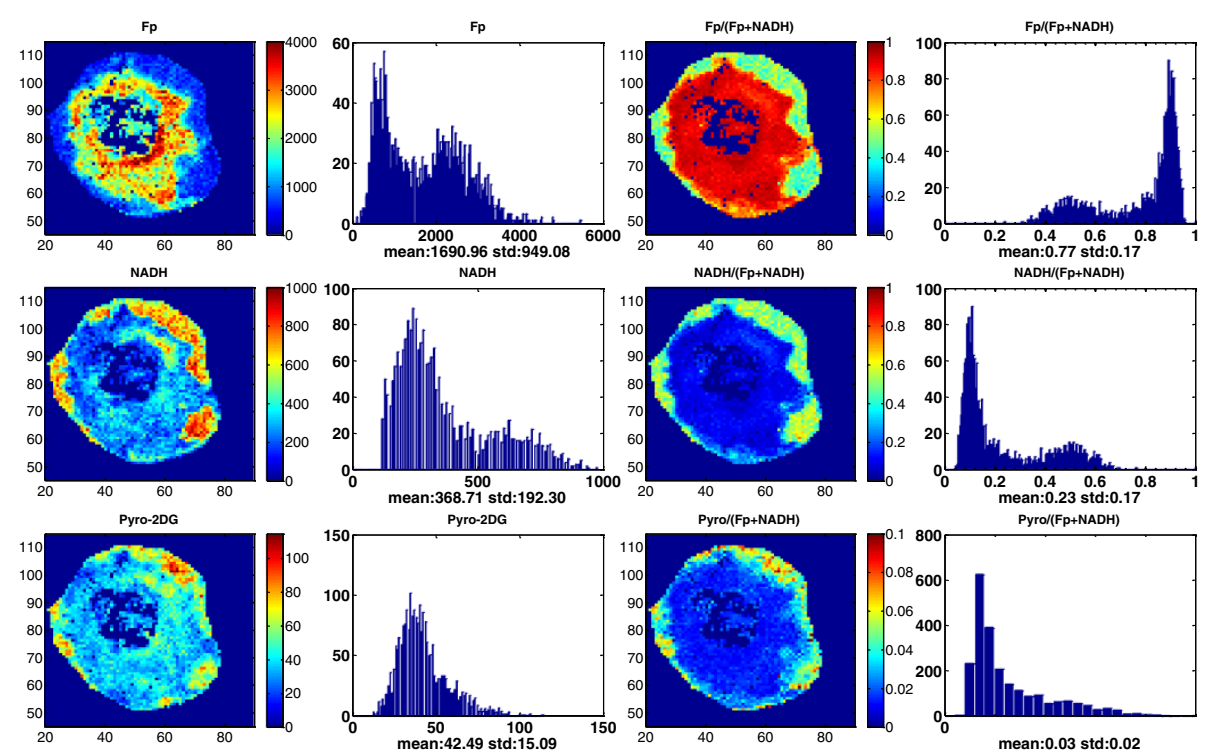

Figure 5 Typical mitochondrial redox state and Pyro-2DG uptake images and their corresponding histograms of a representative section of a large MDA-MB-231 tumor $\left(>10 \mathrm{~mm}, \mathrm{Vol}=766 \mathrm{~mm}^{3}\right)$. See the legend of Figure 4 for notations.

there are islands of cells exhibiting active metabolism indicated by strong NADH, Fp, and Pyro-2DG signals, particularly in the large MCF-7 tumor in Figure 4. Two islands of viable cells in the tumor central region exhibit differential Pyro-2DG uptake as shown by the Pyro-2DG/(Fp + $\mathrm{NADH}$ ) image although their corresponding redox ratios are similar.

In general, all four tumors had oxidized core and reduced peripheral regions mostly corresponding with the lower and the higher pyro-2DG uptake, respectively, and showed a positive correlation of the pyro-2DG signals with NADH or NADH redox ratios in peripheral tissue regions. Figure 6 is a plot of the distribution of the metabolic indices, i.e., the normalized glucose uptake Pyro2DG/(Fp + NADH) versus the NADH redox ratio NADH/ $(\mathrm{Fp}+\mathrm{NADH})$ for the MDA-MB-231 tumor image section displayed in Figure 5. A positive correlation is readily identified between these two indices and the linear regression fit yields a correlation coefficient $R^{2}=0.68$. For the MCF-7 tumor image section in Figure 4, the positive correlation between Pyro-2DG/(Fp + NADH) and NADH/ $(\mathrm{Fp}+\mathrm{NADH})$ is not as obvious and the linear regression fit gives $R^{2}=0.24$. Similar correlations between Pyro-2DG or Pyro-2DG/(Fp + NADH) versus the redox indices $(\mathrm{NADH} /(\mathrm{Fp}+\mathrm{NADH})$ or NADH) were observed in general for all four tumors. Despite the existing correlations, the distributions of the glucose uptake level versus mitochondrial NADH or NADH redox ratio were relatively scattered. On the correlation plots such as in Figure 6, for each normalized pyro-2DG uptake level, there are a number of pixels with different values of redox indices, and vice versa. Since the redox indices such as NADH and the
NADH redox ratio are indicators of the mitochondrial redox state, these observations demonstrate that each pyro-2DG uptake level corresponds to a number of different mitochondrial redox states, and each mitochondrial redox state corresponds to a number of glucose uptake levels. This result indicates that there is a whole variety of cellular metabolic states existing in a breast tumor.

\section{Discussion}

Tumor functional and metabolic heterogeneity has long been recognized. Studies have shown that clinical tumors exhibited more heterogeneous tissue oxygenation

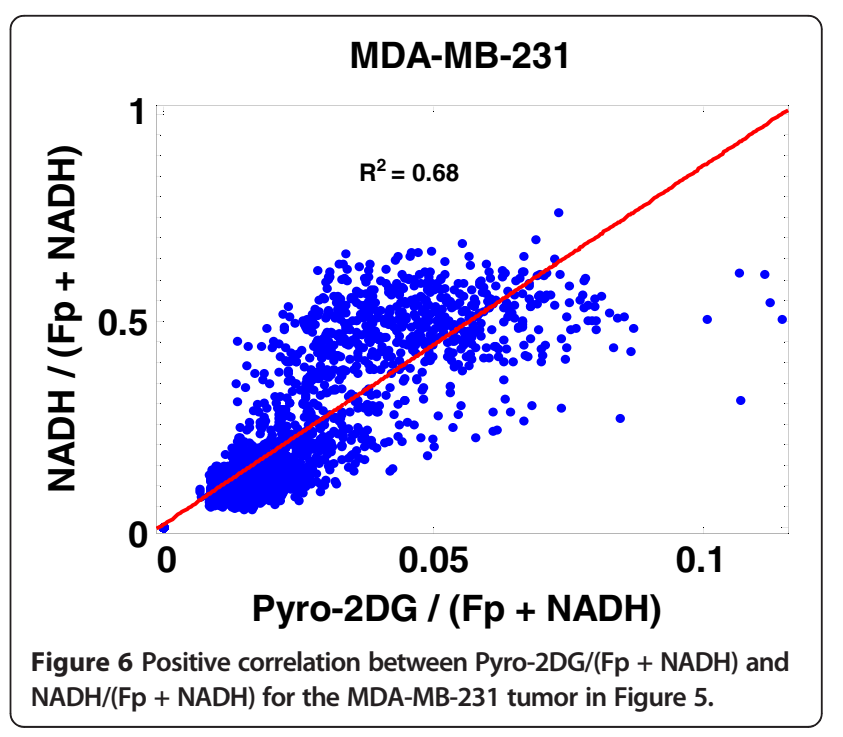


distributions than normal tissue, and the intertumor heterogeneity of tissue oxygenation was larger than intratumor heterogeneity; furthermore, the $\mathrm{pO}_{2}$ variations within breast tumors measured by needle electrodes did not correlate with the measuring site (tumor center versus periphery) (Vaupel et al. 2007; Vaupel et al. 1991). In addition to distinguishing between normal and (pre)malignant tissues (Xu et al. 2013b; Xu et al. 2013a; Xu et al. 2011b), imaging the intratumor heterogeneity of the mitochondrial redox state in tumors may provide useful information for understanding tumor aggressiveness ( $\mathrm{Li}$ 2012). Our previous studies showed that more oxidized mitochondrial redox state in tumor tissue was positively associated with tumor metastatic potential in the melanoma and breast cancer xenograft models ( $\mathrm{Li}$ et al. 2009a; Xu et al. 2010). We also observed that the clinical cancerous breast tissue was much more oxidized and more heterogeneous than the normal tissue (Xu et al. 2013a). The present study shows with great details of the metabolic heterogeneity in breast tumor xenografts and breast tumor specimens, which may be further investigated for developing high resolution biomarkers for clinical applications.

The present study is the first to reveal the 3D distribution of the mitochondrial redox state and Fp and NADH distribution in entire breast tumor xenografts. The three tumor lines we used in the study have the following ascending aggressiveness or invasive/metastatic potential,
MCF-7 < MDA-MB-468 < MDA-MB-231 (Freund et al. 2003). Both MDA-MB-468 and MDA-MB-231 cells are triple-negative (negative for estrogen receptor, Her2/ neu, and progesterone receptor) (Köster et al. 2009; Bartholomeusz et al. 2010; Anders \& Carey 2008) and belong to aggressive tumors. From the redox images, we have observed significantly higher degree of heterogeneity in the more metastatic MDA-MB-231 and MDA-MB-468 tumors and less heterogeneity in the less metastatic MCF-7 tumors $(\leq 10 \mathrm{~mm})$. More quantitative analysis of the redox indices and their correlation with the aggressiveness of these three xenograft models is in progress. We also observed that MCF-7 tumors larger than $10 \mathrm{~mm}$ in diameter became more heterogeneous with distinct core-rim imaging pattern and the oxidized regions having very high Fp redox ratio (Figure 4). This result suggested that the large MCF-7 tumors may have acquired more aggressive features, consistent with the clinical observations that larger tumors tend to be more aggressive/metastatic.

We also investigated the glucose uptake by simultaneously imaging the fluorescence tracer pyro-2DG and the mitochondrial redox state in four large tumors. The results indicated that there were a large variety of cellular metabolic states existing under the complex tumor microenvironment. Although there are intertumor variations, the common patterns are that these tumors all exhibited more oxidized states in tumor central regions and more reduced states in tumor peripherals, and the normalized

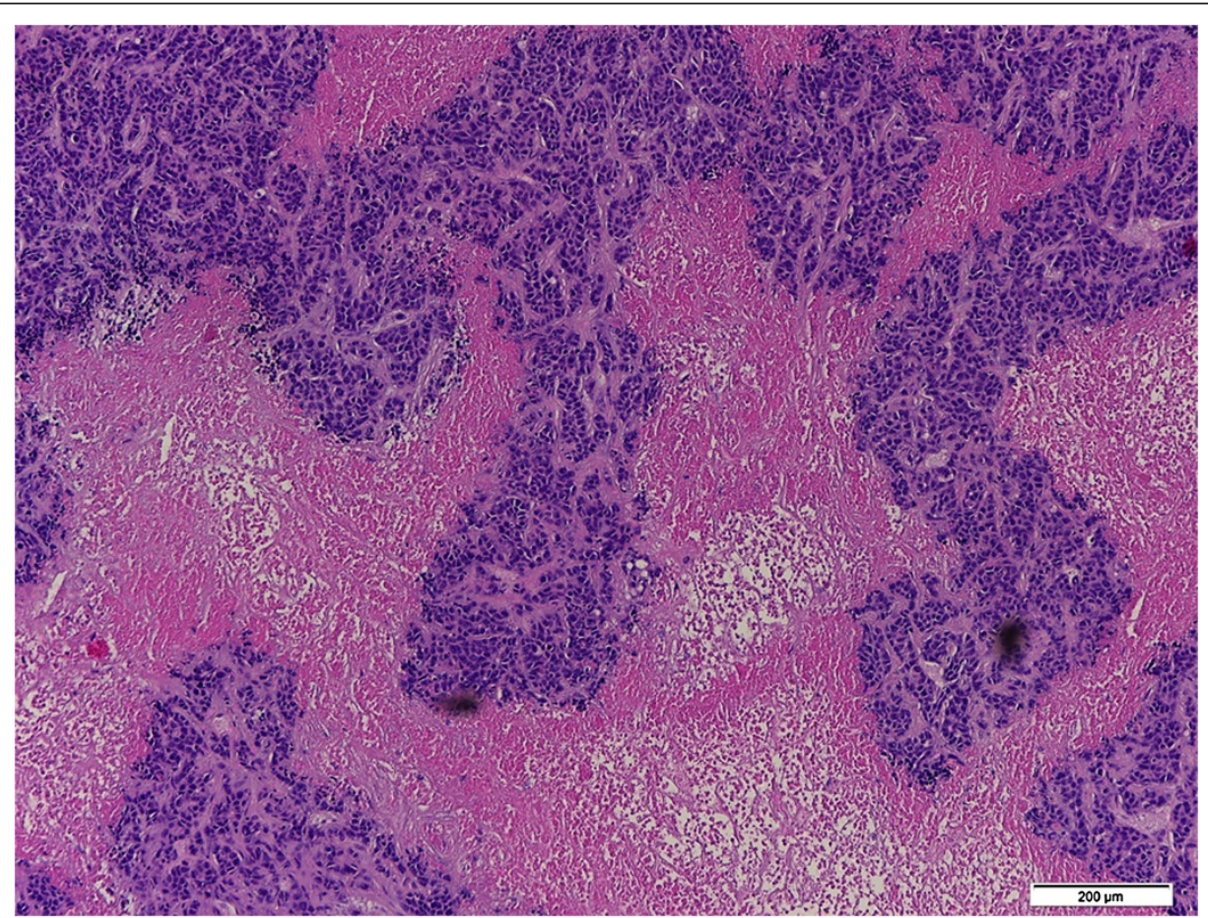

Figure 7 Typical H\&E staining of an area within the necrotic center of a large MCF-7 tumor $(>10 \mathrm{~mm})$ showing islands of viable cells $($ bar $=200 \mu \mathrm{m})$. 
glucose uptake pyro-2DG/(Fp + NADH) grossly positively correlated with $\mathrm{NADH} /(\mathrm{Fp}+\mathrm{NADH})$ in the tumor periphery. Early studies using bioluminescent enzymatic assay demonstrated the heterogeneous spatial distribution of glucose, lactate, and ATP in human breast cancer xenografts in rats (Kallinowski et al. 1988). Heterogeneous uptake of glucose by cancer cells in tumor xenografts was also quantitatively evaluated by radioluminography showing the selective uptake of deoxyglucose for viable cells over necrotic regions (Dearling et al. 2004). Such selectivity was also seen in the present study where tumor peripheral areas have apparently higher Pyro-2DG uptake, corresponding to higher NADH and the NADH redox ratio. This can be explained by the presumably higher perfusion and nutrient delivery and more NADH being metabolically generated in the tumor peripheral areas than in the central regions.

We also observed pronounced Pyro-2DG uptake in the areas inside the necrotic center along with significant $\mathrm{NADH}$ and Fp signals. High glucose uptake and NADH concentration inside the necrotic center indicate that these cells had high metabolic activities. It is likely that these cells were in State 3 where cells had sufficient nutrient resulting in rapid oxidative metabolism (high NADH, $\mathrm{Fp}$ and the Fp redox ratio) rather than in State 2 where cells were nutrient-starved (low NADH, high Fp and the Fp redox ratio) (Chance \& Williams 1955; Chance \& Baltscheffsky 1958; Chance \& Schoener 1966). This also provides a possible answer to our previous question regarding whether the cancer cells in the oxidized regions were in State 2 (starvation) or State 3 (sufficient substrates) (Li et al. 2009a; Xu et al. 2010). According to the present study, it seems that the answer is some were in State 2 and some in State 3.

The metabolic status of the cells in the necrotic center has not received noticeable attentions. The cells in the necrotic area are often considered dead due to limited blood perfusion that usually happens for aggressive tumors in mouse xenografts (Li et al. 2009a; Bradley et al. 2007; Pedley et al. 2001; Ranney et al. 2005). However, "hot" spots with high uptake of radiolabeled glucose were reported to exist in the necrotic areas of tumors in the literature (Dearling et al. 2004). Our previous studies on metastatic melanoma mouse xenografts also found that there was a sizable portion of cells inside the necrotic centers with intact nuclei shown by both the H\&E staining and DAPI staining as well as TUNEL negativity, all of which indicated they were viable (Xu et al. 2010; Xu et al. 2009b). The H\&E staining of breast tumor xenografts also showed islands of cells with intact nuclei in the necrotic/ oxidized regions (Figure 7). It is the redox indices in the oxidized tumor core that readily distinguish between the aggressive and indolent xenografts, better than the whole section average (Li et al. 2009a; Xu et al. 2010). These apparently viable cells in the oxidized cores may provide important clues to understanding tumor aggressiveness or metastatic potential.

\section{Conclusions}

We reported the preliminary results from 3D high resolution mapping of the mitochondrial redox state of three breast cancer lines xenografted in mice, i.e., MCF-7, MDA-MB-468, and MDA-MB-231 with increasing ranks of metastatic potential. We identified significant intertumor and intratumor heterogeneity of the redox state among all xenografts and for the first time revealed the mitochondrial redox state spatial distribution in an entire tumor. Additionally, we showed that high degree of heterogeneity existed in the clinical biopsy samples from the breast cancer patients. We also reported the preliminary results of simultaneous imaging of the mitochondrial redox state and glucose uptake in larger breast cancer xenografts with apparent necrotic centers. We found that the glucose uptake was also heterogeneous (usually higher in tumor periphery) and positively correlates with NADH and the NADH redox ratio in general but not in all regions, and that islands of viable cells existed in tumor necrotic/oxidized centers exhibiting both high glucose uptake and NADH. These results indicated that a variety of metabolic states exist in a breast tumor and the metabolic heterogeneity warrants further investigation for developing potential metabolic biomarkers for clinical applications.

\section{Competing interests}

The authors declare that they have no conflict of interest.

\section{Authors' contributions}

LZL and HNX designed and coordinated the overall study. GZ participated in the study design of simultaneous imaging pyro-2DG and redox state. JT participated in the study design of redox imaging of clinical specimens. JT recruited patients, collected clinical samples, and provided patient clinical information. HNX prepared the animal models, carried out the redox scanning, and performed data analyses. SN provided redox scanning technical support and participated in the data interpretation. HNX and LZL created the data analysis method and wrote the manuscript. All authors read and approved the final manuscript.

This paper is dedicated to the $100^{\text {th }}$ birthday of late Britton Chance (1913-2010) who actively participated in the study design and data interpretation until the last few days of his life.

\section{Acknowledgments}

This work was supported by research grants from Susan G. Komen Research Foundation (KG081069, L.Z. Li) and National Institute of Health (R01CA155348 (L.Z. Li), RR02305, 2U24-CA083105, NCI Cancer Center Support Grant 2-P30-CA-016520-35), and the Linda and Paul Richardson Breast Cancer Research Funds (J. Tchou). We thank Mr. Aron Roxin for synthesizing Pyro2DG,

Dr. Anatoliy Popov and Dr. Zhihong Zhang for their advice on Pyro-2DG solution preparation, Dr. Xiaohong Ma for her assistance in Pyro-2DG tail-vein administration, and Dr. Beamon Agarwal for his assistance in H\&E staining.

\section{Author details}

${ }^{1}$ Molecular Imaging Laboratory, Department of Radiology, Perelman School of Medicine, University of Pennsylvania, Pennsylvania, USA. ${ }^{2}$ Britton Chance 
Laboratory of Redox Imaging, Johnson Research Foundation, Department of Biochemistry and Biophysics, Perelman School of Medicine, University of Pennsylvania, Pennsylvania, USA. ${ }^{3}$ Department of Medical Biophysics, University of Toronto, Toronto, Canada. ${ }^{4}$ Department of Surgery, Perelman School of Medicine, University of Pennsylvania, Pennsylvania, USA. ${ }^{5}$ Rena Rowan Breast Center, Abramson Cancer Center, Perelman School of Medicine, University of Pennsylvania, Pennsylvania, USA.

Received: 14 February 2013 Accepted: 18 February 2013 Published: 28 February 2013

\section{References}

Allred DC, Wu Y, Mao S, Nagtegaal ID, Lee S, Perou CM, Mohsin SK, O'Connell P, Tsimelzon A, Medina D (2008) Ductal carcinoma in situ and the emergence of diversity during breast cancer evolution. Clin Cancer Res 14(2):370-378

Anders C, Carey LA (2008) Understanding and treating triple-negative breast cancer. Oncology (Williston Park) 22(11):1233-1239, discussion 1239-1240, 1243

Bartholomeusz C, Gonzalez-Angulo AM, Kazansky A, Krishnamurthy S, Liu P, Yuan LXH, Yamasaki F, Liu S, Hayashi N, Zhang D, Esteva FJ, Hortobagyi GN, Ueno NT (2010) PEA-15 inhibits tumorigenesis in an MDA-MB-468 triple-negative breast cancer xenograft model through increased cytoplasmic localization of activated extracellular signal-regulated kinase. Clin Cancer Res 16(6):1802-1811

Bradley DP, Tessier JJ, Ashton SE, Waterton JC, Wilson Z, Worthington PL, Ryan AJ (2007) Correlation of MRI biomarkers with tumor necrosis in Hras5 tumor xenograft in athymic rats. Neoplasia 9(5):382-391

Cairns RA, Harris IS, Mak TW (2011) Regulation of cancer cell metabolism. Nat Rev Cancer 11(2):85

Chance B, Baltscheffsky H (1958) Respiratory enzymes in oxidative phosphorylation. VII. Binding of intramitochondrial reduced pyridine nucleotide. J Biol Chem 233(3):736-739

Chance B, Schoener B (1966) High and low energy states of cytochromes. J Biol Chem 241(20):4567-4573

Chance B, Williams GR (1955) Simple and rapid assay of oxidative phosphorylation. Nature 175(4469):1120-1121

Chance B, Schoener B, Oshino R, Itshak F, Nakase Y (1979) Oxidation-reduction ratio studies of mitochondria in freeze-trapped samples. NADH and flavoprotein fluorescence signals. J Biol Chem 254(11):4764-4771

Dearling JLJ, Flynn AA, Sutcliffe-Goulden J, Petrie IA, Boden R, Green AJ, Boxer GM, Begent RHJ, Pedley RB (2004) Analysis of the regional uptake of radiolabeled deoxyglucose analogs in human tumor xenografts. J Nucl Med 45(1):101-107

Freund A, Chauveau C, Brouillet JP, Lucas A, Lacroix M, Licznar A, Vignon F, Lazennec G (2003) IL-8 expression and its possible relationship with estrogen-receptor-negative status of breast cancer cells. Oncogene 22(2):256-265

Gu Y, Qian Z, Chen J, Blessington D, Ramanujam N, Chance B (2002) High-resolution three-dimensional scanning optical image system for intrinsic and extrinsic contrast agents in tissue. Rev Sci Instrum 73(1):172-178

Hanahan D, Weinberg RA (2011) Hallmarks of cancer: the next generation. Cell 144(5):646-674

Heppner GH (1984) Tumor heterogeneity. Cancer Res 44(6):2259-2265

Heppner GH, Miller FR (1998) The cellular basis of tumor progression. Int Rev Cytol 177:1-56

Kallinowski F, Vaupel P, Runkel S, Berg G, Fortmeyer HP, Baessler KH, Wagner K, Mueller-Klieser W, Walenta S (1988) Glucose uptake, lactate release, ketone body turnover, metabolic micromilieu, and pH distributions in human breast cancer xenografts in nude rats. Cancer Res 48(24 Pt 1):7264-7272

Koppenol WH, Bounds PL, Dang CV (2011) Otto Warburg's contributions to current concepts of cancer metabolism. Nat Rev Cancer 11(5):325

Köster F, Engel J, Schally A, Hönig A, Schröer A, Seitz S, Hohla F, Ortmann O, Diedrich K, Buchholz S (2009) Triple-negative breast cancers express receptors for growth hormone-releasing hormone $(\mathrm{GHRH})$ and respond to GHRH antagonists with growth inhibition. Breast Cancer Res Treat 116(2):273

Li LZ (2012) Imaging mitochondrial redox potential and its possible link to tumor metastatic potential. J Bioenerg Biomembr 44:645-653

Li LZ, Zhou R, Xu HN, Moon L, Zhong T, Kim EJ, Qiao H, Reddy R, Leeper D, Chance B, Glickson JD (2009a) Quantitative magnetic resonance and optical imaging biomarkers of melanoma metastatic potential. Proc Natl Acad Sci U S A 106(16):6608-6613
Li LZ, Xu HN, Ranji M, Nioka S, Chance B (2009b) Mitochondrial redox imaging for cancer diagnostic and therapeutic studies. J Innov Optical Health Sci 2:325-341

Park SY, Lee HE, Li H, Shipitsin M, Gelman R, Polyak K (2010) Heterogeneity for stem cell-related markers according to tumor subtype and histologic stage in breast cancer. Clin Cancer Res 16(3):876-887

Pedley RB, Hill SA, Boxer GM, Flynn AA, Boden R, Watson R, Dearling J, Chaplin DJ, Begent RH (2001) Eradication of colorectal xenografts by combined radioimmunotherapy and combretastatin a-4 3-O-phosphate. Cancer Res 61(12):4716-4722

Quistorff B, Haselgrove JC, Chance B (1985) High spatial resolution readout of 3-D metabolic organ structure: an automated, low-temperature redox ratioscanning instrument. Anal Biochem 148(2):389-400

Ranney D, Antich P, Dadey E, Mason R, Kulkarni P, Singh O, Chen H, Constantanescu A, Parkey R (2005) Dermatan carriers for neovascular transport targeting, deep tumor penetration and improved therapy. J Control Release 109(1-3):222-235

Thompson CB (2009) Metabolic enzymes as oncogenes or tumor suppressors. N Engl J Med 360(8):813-815

Vander Heiden MG, Cantley LC, Thompson CB (2009) Understanding the Warburg effect: the metabolic requirements of cell proliferation. Science 324(5930):1029-1033

Vaupel P, Schlenger K, Knoop C, Hockel M (1991) Oxygenation of human tumors: evaluation of tissue oxygen distribution in breast cancers by computerized $\mathrm{O} 2$ tension measurements. Cancer Res 51(12):3316-3322

Vaupel P, Hockel M, Mayer A (2007) Detection and characterization of tumor hypoxia using pO2 histography. Antioxid Redox Signal 9(8):1221-1235

Xu HN, Wu B, Nioka S, Chance B, Li LZ (2009a) Calibration of redox scanning for tissue samples. Proc of SPIE 7174:71742F1-71742F8

Xu HN, Zhou R, Nioka S, Chance B, Glickson JD, Li LZ (2009b) Histological basis of MR/optical imaging of human melanoma mouse xenografts spanning a range of metastatic potentials. Adv Exp Med Biol 645:247-253

Xu HN, Nioka S, Glickson JD, Chance B, Li LZ (2010) Quantitative mitochondrial redox imaging of breast cancer metastatic potential. J Biomed Opt 15(3):036010

Xu HN, Nioka S, Chance B, Zheng G, Li LZ (2011a) High-resolution simultaneous mapping of mitochondrial redox state and glucose uptake in human breast tumor xenografts. Adv Exp Med Biol 737:175-179

Xu HN, Nioka S, Chance B, Li LZ (2011b) Heterogeneity of mitochondrial redox state in premalignant pancreas in a PTEN null transgenic mouse model. Adv Exp Med Biol 701:207-213

Xu HN, Nioka S, Chance B, Li LZ (2012) 3-D high-resolution mapping of the heterogeneity in mitochondrial redox state of human breast tumor xenografts. Adv Exp Med Biol 737:169-174

Xu HN, Tchou J, Chance B, Li LZ (2013a) Imaging the redox states of human breast cancer core biopsies. Adv Exp Med Biol 765:343-349

Xu HN, Nioka S, Chance B, Li LZ (2013b) Imaging heterogeneity in the mitochondrial redox state of premalignant pancreas in the pancreas-specific PTEN-null transgenic mouse model. Biomarker Res 1:6

Zhang M, Zhang Z, Blessington D, Li H, Busch TM, Madrak V, Miles J, Chance B, Glickson JD, Zheng G (2003) Pyropheophorbide 2-deoxyglucosamide: a new photosensitizer targeting glucose transporters. Bioconjug Chem 14(4):709-714

Zhang ZH, Li H, Liu Q, Zhou LL, Zhang M, Luo QM, Glickson J, Chance B, Zheng $G$ (2004) Metabolic imaging of tumors using intrinsic and extrinsic fluorescent markers. Biosens Bioelectron 20(3):643-650

doi:10.1186/2193-1801-2-73

Cite this article as: Xu et al.: Characterizing the metabolic heterogeneity in human breast cancer xenografts by $3 \mathrm{D}$ high resolution fluorescence imaging. SpringerPlus 2013 2:73. 